Check for updates

Cite this: RSC Adv., 2018, 8, 18334

Received 8th March 2018

Accepted 30th April 2018

DOI: $10.1039 / \mathrm{c} 8 \mathrm{ra02058b}$

rsc.li/rsc-advances

\section{Enhancement of thermoelectric performance of PEDOT:PSS films by post-treatment with a superacid}

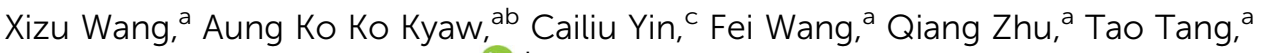 \\ Phang In Yee ${ }^{a}$ and Jianwei Xu (iD *a
}

Several methods such as the addition of a polar solvent, an acid as well as various post-treatments have been used to improve the thermoelectric performance of conductive poly $(3,4$ ethylenedioxythiophene):polystyrene sulfonate (PEDOT:PSS) films. This paper reports a method using a superacid, trifluoromethanesulfonic acid, in methanol to treat PEDO:PSS films to improve their thermoelectric performance. Treatment of PEDOT:PSS films with this superacid in methanol leads to a significant increase in electrical conductivity from 0.7 to $2980 \mathrm{~S} \mathrm{~cm}^{-1}$ together with a moderate increase in Seebeck coefficient from 17.6 to $21.9 \mu \mathrm{V} \mathrm{K}$, giving a power factor of $142 \mu \mathrm{W} \mathrm{m} \mathrm{m}^{-1} \mathrm{~K}^{-2}$, one of the highest values reported in the literature for conductive polymers. The figure of merit ( $Z T)$ value is estimated to be 0.19 under optimized conditions. The enhancement of thermoelectric performance, particularly the increase in both electrical conductivity and Seebeck coefficient, is due to the removal of the insulating component and polymer chain realignment giving in turn a denser packing of the conductive PEDOT polymer chains. This post-treatment method would offer an alternative way to improve the thermoelectric performance.

\section{Introduction}

Thermoelectric (TE) materials are used as a thermal power generator to convert heat directly into electrical energy or as a solid state Peltier cooler. ${ }^{1}$ Organic based semiconductors are emerging as next potential TE materials that offer several advantages such as low cost, light weight, flexibility and solution-process fabrication although its efficiency is still lower than that of inorganic counterparts such as $\mathrm{Bi}_{2} \mathrm{Te}_{3}$ and $\mathrm{Sb}_{2} \mathrm{Te}_{3} \cdot{ }^{2-5}$ In addition, organic materials with intrinsically low thermal conductivity, which is over 2 to 3 orders of magnitude lower than that of commercial inorganic materials, make them potential candidates for high performance TE applications. Polymeric TE materials such as poly(thienothiophene), ${ }^{6}$ polyaniline, ${ }^{7}$ poly(3,4-ethylenedioxythiophene) (PEDOT), ${ }^{8}$ polyacetylene, ${ }^{9}$ and poly(3-hexylthiophene) have been investigated. ${ }^{10}$ Among them, PEDOT:PSS is considered as an unavoidable candidate. The uniformly dispersed aqueous solution of conductive PEDOT polymer with PSS polymer as both stabilizer and counterion is solution-processable and the corresponding conductive film is flexible. What is more, theoretical simulation

${ }^{a}$ Institute of Materials Research and Engineering, A*STAR, 2 Fusionopolis Way, Innovis, \#08-03, 138634, Singapore.E-mail: jw-xu@imre.a-star.edu.sg

${ }^{b}$ Department of Electrical and Electronic Engineering, Southern University of Science and Technology, Shenzhen 518055, P. R. China

${ }^{c}$ College of Science, Guangxi University for Nationalities, Nanning, 530006, China also indicated that the TE performance of PEDOT:PSS could compete with commercial inorganic TE materials. ${ }^{\mathbf{1 1}}$

A dimensionless thermoelectric figure of merit $(Z T)$ is calculated in terms of $Z T=S^{2} \sigma T / \kappa$, where $S, \sigma, T$ and $\kappa$ are the Seebeck coefficient, electrical conductivity, absolute temperature and thermal conductivity, respectively. The power factor $S^{2} \sigma$, which is defined as the product of electric conductivity $(\sigma)$ and square of the Seebeck coefficient $\left(S^{2}\right)$, is a key parameter to measure the usefulness of a given thermoelectric material. The flexible PEDOT:PSS thin films show great potential for TE applications at room temperature, ${ }^{\mathbf{1 2 , 1 3}}$ but the low Seebeck coefficient as well as low electrical conductivity of pristine PEDOT are not desirable to achieve a high power factor for expected TE application. ${ }^{8}$ In general, addition of a polar solvent, as an additive, such as dimethylsulfoxide (DMSO), ethylene glycol or methanol, tremendously increases the electrical conductivity due to the realignment of conducive PEDOT chains. ${ }^{\mathbf{1 4 - 1 6}}$ In contrast, post-treatment of PEDOT:PSS thin films with a mineral acid improves the electrical conductivity and Seebeck coefficient simultaneously by changing both the chain conformation and oxidation level of PEDOT, as a result, maximizing the power factor. ${ }^{17,18}$ The polar solvent, inorganic acid, base or salt solutions are reported to weaken the interactions between PEDOT and PSS in different ways, resulting in easier removal of the insulating part (PSS), and hence enhancing its electrical conductivity. ${ }^{19-26}$ 
To achieve a higher power factor, TE materials are required to have both high electrical conductivity and a large Seebeck coefficient. However, the conflicting interrelation of the Seebeck coefficient and electrical conductivity imposes limitations on the optimization of the power factor value. Therefore, the ultrahigh conductivity of PEDOT:PSS ${ }^{18,27}$ does not guarantee to achieve a high power factor because of the trade-off between the electrical conductivity and the Seebeck coefficient. ${ }^{28}$ Another disadvantage is the PEDOT:PSS thin film is relatively easily damaged and is also unstable during inorganic acid treatment since part of polymer dissolves in a water-based acid. However, using anhydrous or pure organic acid treatment is very likely to keep better thermal performance and humidity stability while ensuring the high electrical conductivity of PEDOT:PSS thin film. In addition, a recent study shows that iron(III) trifluoromethanesulfonate used in PEDOT-based polymer synthesis exhibited great metallic behaviour with induced high oxidation state in conductive polymers by replacing the role of PSS polymer, giving us implication that fluorine-bearing acids may be potential to modulate $\mathrm{TE}$ performance of polymer materials. $^{32}$

In our work, treatment using an anhydrous trifluoromethanesulfonic acid and methanol (TFMS-MeOH; v/v = $1: 10)$ as a post-treatment solvent is considered to dedope pristine PEDOT:PSS to improve TE performance of polymer thin film. TFMS has a very low $\mathrm{p} K_{\mathrm{a}}$ value of -14.7 , which is much smaller than those of inorganic counterparts $\mathrm{H}_{2} \mathrm{SO}_{4}(-3,2)$ and $\mathrm{HCl}(-7)$. TFMS is considered as a superacid and it is thermally and chemically stable. Unlike other acids, the conjugate base of TFMS $\left(\mathrm{CF}_{3} \mathrm{SO}_{3}{ }^{-}\right)$does not behave as a nucleophile, and thus TFMS is expected to be more useful in protonation than other acids. Therefore, TFMS is likely able to more effectively reduce the PSS concentration than other chemicals used for posttreatment due to much stronger acidity than any other acids. In conjunction with structural rearrangement and high dense packing of the PEDOT induced by the reduction of PSS, TFMS$\mathrm{MeOH}$ is anticipated to cause significant conductivity improvement. The process by the combination of two types of solvents involving in the post-treatment of PEDOT:PSS nanothin film would offer a better alternative way to optimize thermoelectric properties of conductive polymers than single chemical post-treatment.

\section{Experimental}

\section{Materials and fabrication}

The PEDOT:PSS aqueous solution (PH1000, Heraeus Clevios) was purchased from Heraeus, Germany. Other chemicals were purchased from Sigma-Aldrich and were used without further purification. The glass substrates were pre-cleaned successively with detergent (30 min), de-ionized (DI) water (30 min), acetone (10 $\mathrm{min})$ and IPA (10 $\mathrm{min})$ with ultrasonic and finally treated with UV-ozone for $10 \mathrm{~min}$. The PEDOT:PSS films were prepared by spin-coating the PEDOT:PSS solution onto $2.5 \times$ $2.5 \mathrm{~cm}^{2}$ glass substrates. After spin-coating, the PEDOT:PSS films were dried at $120{ }^{\circ} \mathrm{C}$ on a hot plate for $10 \mathrm{~min}$. A mixture of trifluoromethanesulfonic acid and methanol $(\mathrm{v} / \mathrm{v}=1: 10)$ or pure methanol were used to treat PEDOT:PSS films by dropping $200 \mu \mathrm{L}$ liquid onto a PEDOT:PSS thin film on the hot plate at $130{ }^{\circ} \mathrm{C}$. The film was dried for about $30 \mathrm{~min}$, and then the dried films were washed by dropping it into methanol for three times. Finally, the polymer films were dried again at $130{ }^{\circ} \mathrm{C}$ for about $2 \mathrm{~min}$ and then was cooled down to room temperature for next use. Following the similar process as described above, DMSO-treated film was prepared using a mixtures of $5 \%$ vol DMSO and PEDOT:PSS solution.

\section{Instrumentations}

The thicknesses of the coating films were measured by the KLA Tencor P-16+ Surface Profiler. The electrical conductivity of coating films was measured by Loresta-GP MCP-T600 (Mitsubishi Low Resistivity Meter) at ambient temperature and relative humidity (RH) 50\%. The Seebeck coefficient was measured by a custom-made system with an $\mathrm{S}$ A Peltier heater $(298 \mathrm{~K}+\Delta T)$ and a Peltier cooler ( $298 \mathrm{~K}$ ) used to apply and vary the temperature gradients on two ends of the coating film and induce a thermal voltage. Two microthermocouples (diameter 0.20 $\mathrm{mm}$ ) were placed on the coating film alongside two electrodes which were connected to a Keithley 2400 source meter. The Seebeck coefficient value was then derived from a linear fit of the measured $\Delta V$ versus $\Delta T$ graph.

The absorption spectra were obtained on an UV-Vis-NIR spectrophotometer (Shimadzu UV-Vis spectrophotometer UV3600). The PEDOT:PSS films for the absorption spectra were fabricated using a spin coating method on quartz substrates and the film thickness was estimated to be around $50 \mathrm{~nm}$. X-ray Photoelectron Spectrometer (XPS) measurement was performed on a Theta Probe Angle-Resolved X-ray Photoelectron Spectrometer (ARXPS) System (Thermo Scientific) at a base pressure of $1 \times 10^{-9}$ torr and a step size of $0.1 \mathrm{eV}$ using monochromated, micro-focused Al K-alpha X-ray photons ( $h \nu=1486.6 \mathrm{eV})$. The curve fitting and linear background subtraction were carried out using the Avantage software. The Raman spectra were collected using micro-Raman spectra with a laser wavelength of $472 \mathrm{~nm}$ $(2.63 \mathrm{eV})$, a laser beam spot size of $0.5 \mu \mathrm{m}$ and an accumulation time of $10 \mathrm{~s}$. Atomic Force Microscopy (AFM) images were obtained on a Bruker Dimension Icon Atomic Force Microscope using the tapping mode. The mobility and carrier concentration were determined on a Hall effect measurement system using a van der Pauw method. X-ray diffraction experiments were conducted on a Bruker AXS (D8 ADVANCE GADDS) X-ray diffractometer with $\mathrm{Cu} \mathrm{K} \alpha$ radiation $(l=1.54 \AA)$; the beam diameter for this instrument can range from 0.05 to $0.8 \mathrm{~mm}$. The thermal conductivity $\kappa$ was calculated using the formula $\kappa=$ $b^{2} / c \rho$ where thermal effusivity $(b)$, specific heat capacity $(c)$ and density $(\rho)$ are measured independently. The thermal effusivity was measured by using the pulsed light heating thermoreflectance method with a front heating and front detection configuration (NanoTR from NETZSCH). The effusivity values are derived from the curve fitting of thermoreflectance signal, using the specific heat capacity and density of Al film given as $897 \mathrm{~J} \mathrm{~kg}^{-1} \mathrm{~K}^{-1}$ and $2700 \mathrm{~kg} \mathrm{~m}^{-3}$, respectively. The specific heat capacity was measured by Differential Scanning Calorimeter 


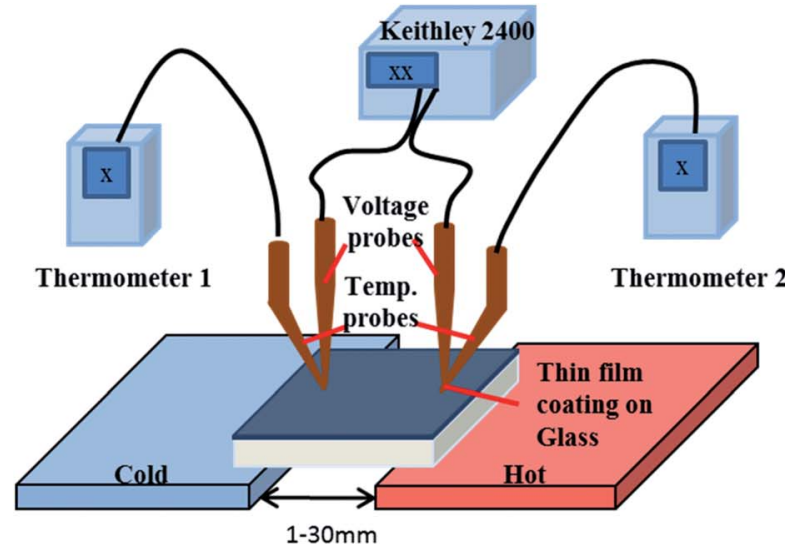

(a)

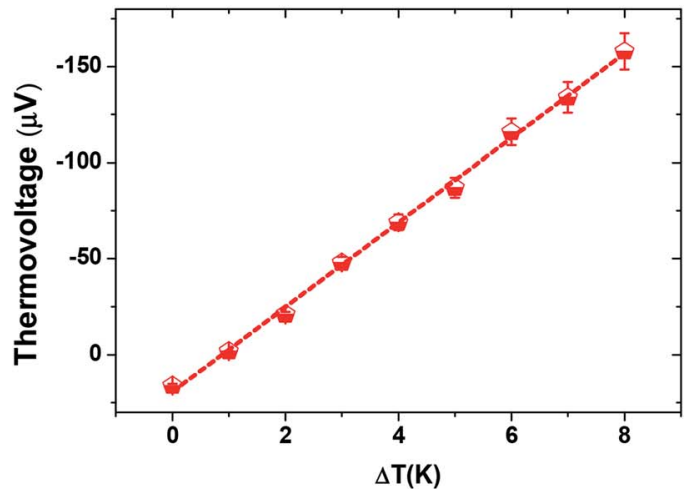

(b)

Fig. 1 Thermoelectric characterization of the PEDOT: thin films. (a) Schematic diagram of the measurement setup. The Peltier plates are used to heat and cool the two sides of the substrate and $\mathrm{K}$ type thermal couples are used to measure the temperature profile of surface. The voltage generated between two of the metal contacts is measured using probes and a Keithley 2400 source meter. (b) Measured voltage as a function of the applied temperature difference of pristine PEDOT:PSS thin film from room temperature, the Seebeck coefficient is extracted from the linear fit.

(Mettler Toledo). The density was estimated based on the mass and volume of the film.

\section{Results and discussion}

The Seebeck coefficient was measured by the custom-made measurement system in shown Fig. 1(a)..$^{11,12,24,35-37}$ The thermovoltage characteristics, Seebeck coefficient of pristine PEDOT:PSS thin films were derived by linearly fitting $\Delta V$ versus the $\Delta T$ at room temperature (Fig. 1(b)). The fluctuation in the Seebeck coefficient was typically within an error range of about $5-10 \%$. The thermal physical performance of the PEDOT:PSS thin film with and without treatment, such as the electrical conductivity and Seebeck coefficient was as shown in Fig. 2. The conductivity and Seebeck coefficient of pristine PEDOT:PSS film were similar to these reported by majority of groups. ${ }^{12,22-25}$ All the thermal characterization was conducted at ambient conditions $\left(21^{\circ} \mathrm{C}\right.$ and $\left.60-70 \% \mathrm{RH}\right)$. The electrical conductivity of thin film was $0.7,880,920,2980 \mathrm{~S} \mathrm{~cm}^{-1}$ for pristine, mixing with $5 \%$
DMSO, methanol post-treatment and TFMS-MeOH posttreatment, respectively. As shown in Fig. 2(a), the highest electrical conductivity achieved by TFMS-MeOH post-treatment is approximately close to $3000 \mathrm{~S} \mathrm{~cm}^{-1}$, indicating that the cosolvent based on anhydrous trifluoromethanesulfonic acid and methanol can enhance the electrical conductivity of PEDOT:PSS to an ultra-high level. ${ }^{18,27}$ Meanwhile, we observed the thickness of the thin film for pristine, mixing with $5 \%$ DMSO, methanol post-treatment and TFMS-MeOH posttreatment are 50, 45, 35 and $25 \mathrm{~nm}$, respectively. Clearly, the processing method that gives a largest reduction in thickness results in a highest conductivity. The highest electrical conductivity could be justified in term of a two-step process between TFMS-MeOH co-solvent and PEDOT:PSS film: the reaction between $\mathrm{CF}_{3} \mathrm{SO}_{3} \mathrm{H}$ and PSS-polyanions produces nonnucleophilic $\mathrm{CF}_{3} \mathrm{SO}_{3}{ }^{-}$anion and $\mathrm{PSSH}$ polyacid, and then the resulting PSSH polyacid subsequently dissolves in methanol and eventually is washed away by methanol. The Seebeck
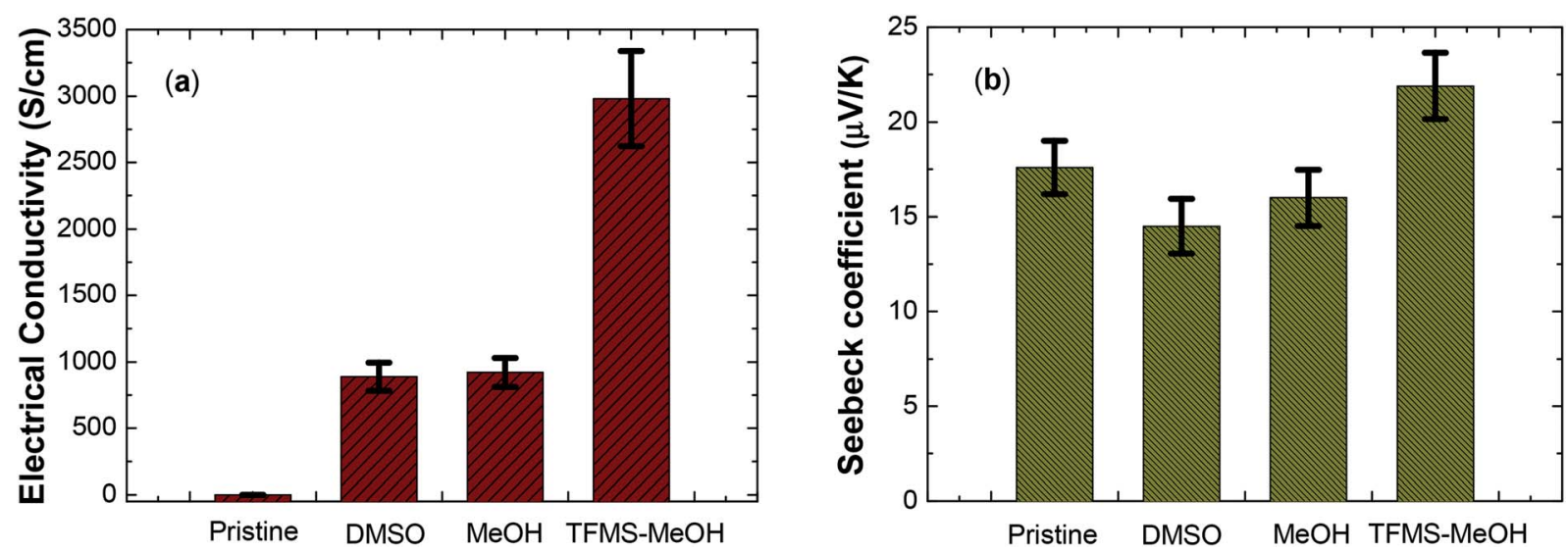

Fig. 2 Electrical conductivity (a) and Seebeck coefficient (b) of PEDOT:PSS thin films with different solvent treatment. 

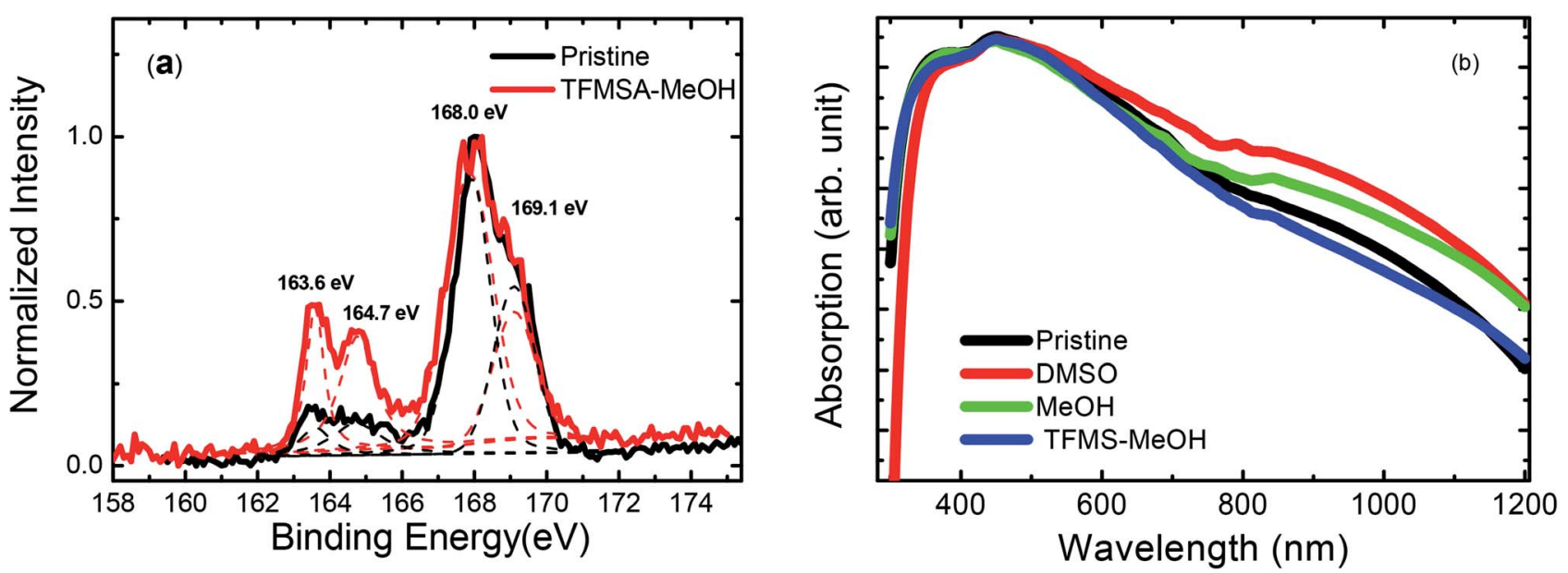

Fig. 3 (a) The S 2p XPS spectra of glass substrate, pristine and TFMS-MeOH treated PEDOT:PSS thin film. (b) The absorption spectra of PEDOT:PSS thin films with and without treatment.

coefficient decreased from $17.6 \mu \mathrm{V} \mathrm{K}{ }^{-1}$ for pristine PEDOT:PSS film to $14.5 \mu \mathrm{V} \mathrm{K}^{-1}$ for PEDOT:PSS nanofilm prepared by mixing with 5\% DMSO. Similarly, pristine PEDOT:PSS film post-treated methanol only gave a comparable Seebeck coefficient of $16 \mu \mathrm{V}$ $\mathrm{K}^{-1}$. The decrease in the Seebeck coefficient after organic solvent treatment may be partially due to increase in carrier concentration in air with humidity condition, ${ }^{40}$ while the concentration of PSS in the PEDOT:PSS nanofilm still remains moderately reduced in comparison with PEDOT:PSS film treated with TFMS-MeOH. The Seebeck coefficient of the thin film after TFMS-MeOH treatment was slightly higher than that of the pristine film. Together with the remarkable increase in electrical conductivity and moderate enhancement in the Seebeck coefficient, the power factor of TFMS-MeOH treated PEDOT:PSS nanofilm remarkably increased from 0.022 to 143 $\mu \mathrm{W} \mathrm{m}{ }^{-1} \mathrm{~K}^{-2}$, which is higher than 18.5 and $23.6 \mu \mathrm{W} \mathrm{m}{ }^{-1} \mathrm{~K}^{-2}$ for DMSO and $\mathrm{MeOH}$ treated films, respectively. Different from PEDO:PSS using DMSO as a dopant, our TFMS-MeOH posttreatment showed the improvement of the Seebeck coefficient and electrical conductivity, simultaneously. This is due to the fact that the lightly doped conductive polymer in general exhibits a higher Seebeck coefficient than the heavily doped conductive polymer. ${ }^{5}$ Therefore, the above observation implies that the combination of TFMS and methanol post-treatment is much more effective than single pure polar solvent or acid treatment to improve the thermoelectric properties of PEDOT:PSS nanothin films.

The treatment of PEDOT:PSS thin film with co-solvent has shown to increase conductivity significantly and reduce PSS content as evidenced by X-ray photoelectron spectra (XPS) in

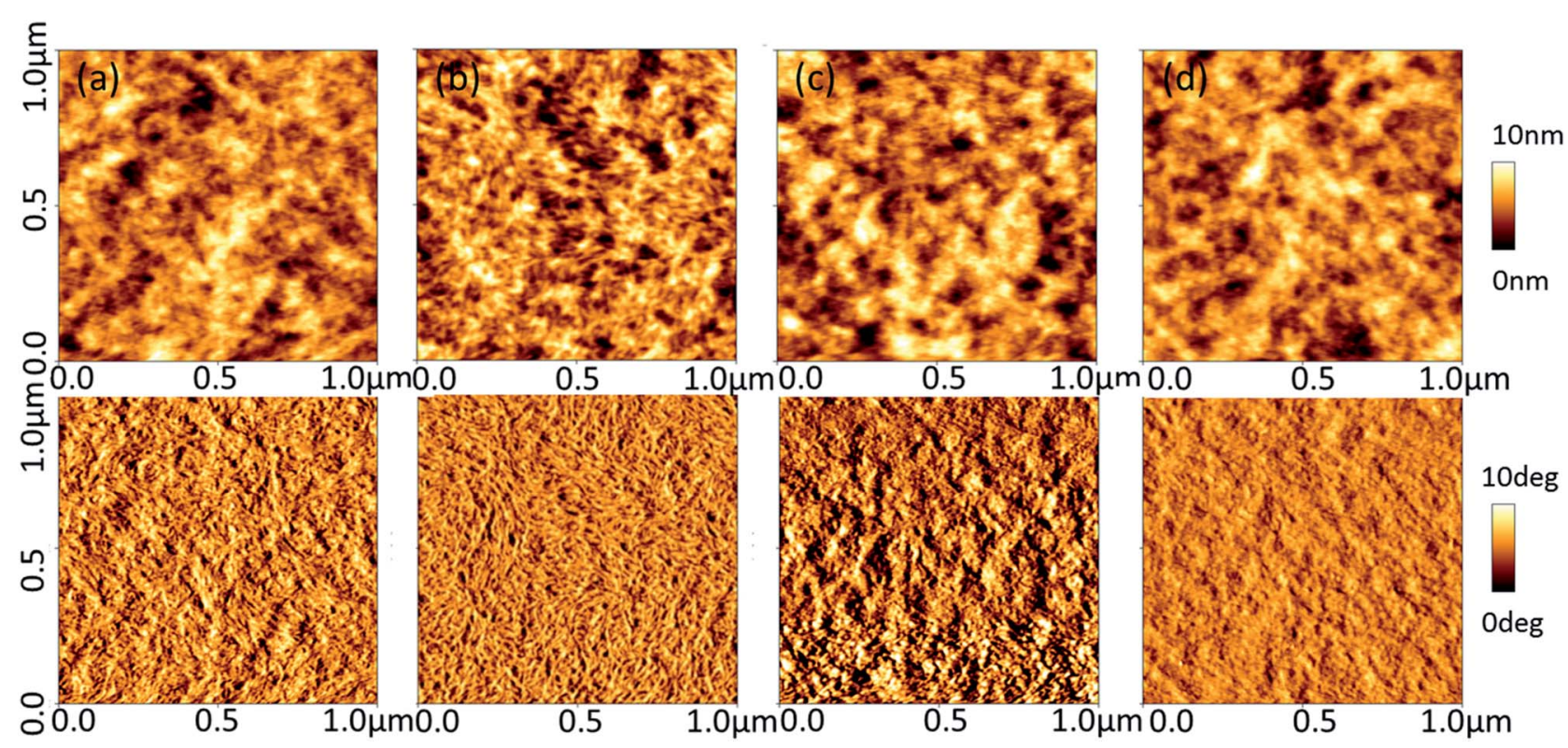

Fig. 4 The AFM surface morphology of PEDOT:PSS thin films with different solvent treatment: (a) pristine, (b) DMSO treatment, (c) MeOH treatment, (d) TFMSA-MeOH treatment. The upper and lower row images are height images and phase images, respectively. 


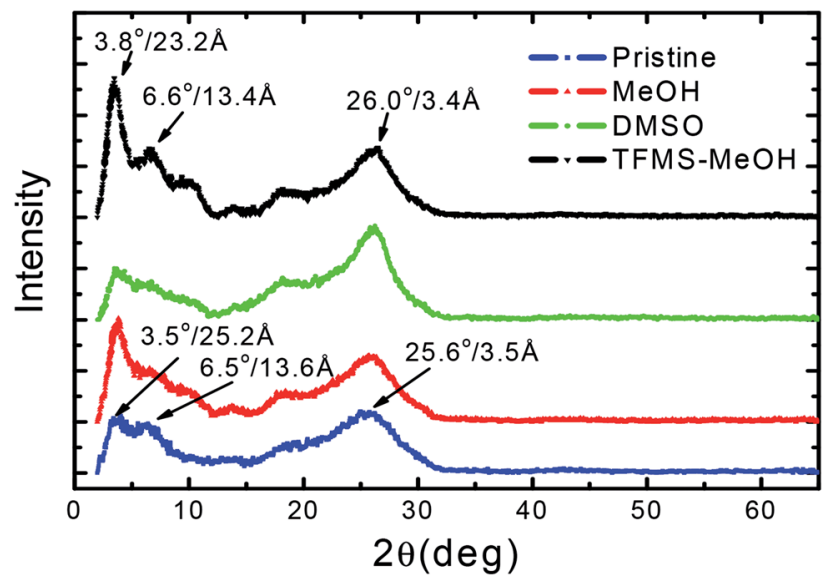

Fig. 5 X-ray diffraction (XRD) patterns of PEDOT:PSS thin films pristine and with different solvent treatment.

Fig. 3(a). The two XPS bands for 168.0 and $169.1 \mathrm{eV}$ are the $\mathrm{S} 2 \mathrm{p}$ band of the sulphur signal from PSS, whereas the two XPS bands for 163.6 and $164.7 \mathrm{eV}$ are the $\mathrm{S} 2 \mathrm{p}$ band of the sulphur signal from PEDOT..$^{\mathbf{1 8 2 9 , 3 0}}$ The $\mathrm{S} 2 \mathrm{p}$ XPS intensity ratio of PEDOT to PSS saliently increased after the TFMS-MeOH treatment, indicating the decrease of some polystyrene sulfonic acid (PSSH) chains in the PEDOT:PSS film. In addition, the C/S molar ratio of XPS spectra changed from 5.8 to 4.2 after the TFMS$\mathrm{MeOH}$ treatment, being consistent with the removal of part of PSSH from PEDOT:PSS film. ${ }^{18,31}$ Fig. $3(\mathrm{~b})$ shows the absorption spectra of pristine, DMSO, MeOH and TFMS-MeOH treated PEDOT:PSS films, respectively, over the wavelength of 300$1200 \mathrm{~nm}$. PEDOT:PSS film treated with TFMS-MeOH exhibited a comparable absorption profile to the pristine film, but it had slightly weaker absorption in the range of 700-1200 nm, which corresponds to the absorption of polaron, than PEDOT:PSS films treated with DMSO and $\mathrm{MeOH}$.

To decipher the effect of treatment on the film morphologies, the atomic force microscopy (AFM) was used to image pristine and treated films. As shown in Fig. 4, the roughness of

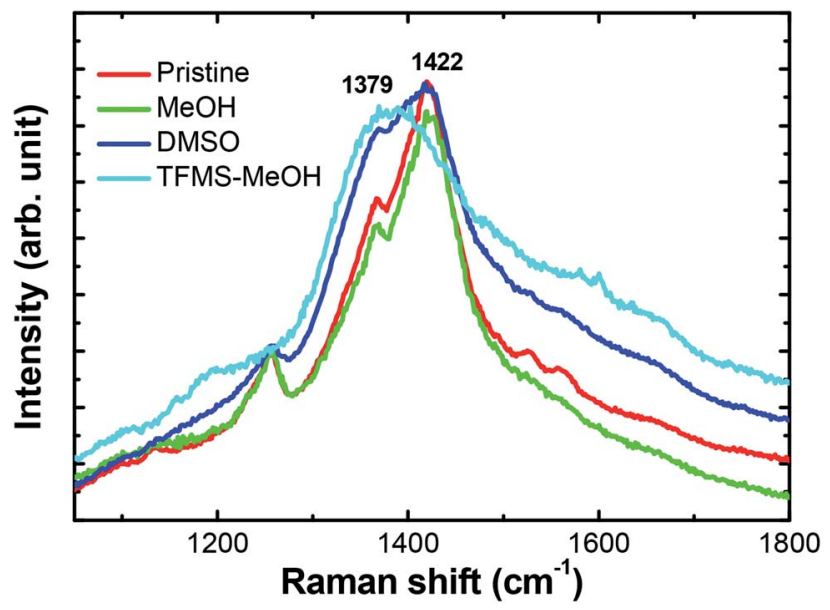

Fig. 6 Raman spectra of the pristine, $\mathrm{MeOH}$ treated, DMSO doping and TFMSA-MeOH treated PEDOT:PSS films.

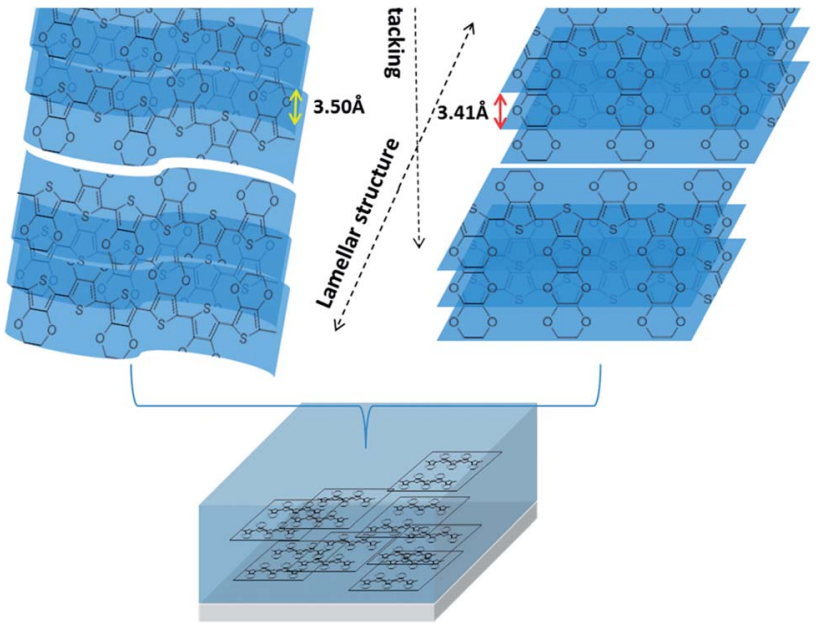

Fig. 7 The diagram of molecular structure arrangement of PEDOT film before and after TFMS-MeOH treatment.

the thin film is $1.61,1.72,1.76,1.58 \mathrm{~nm}$ for without treatment, 5\% DMSO mixing, methanol post-treatment and TFMS-MeOH post-treatment, respectively. The AFM phase images usually show better phase separation between two polymer chains ${ }^{38,39}$ in PEDOT:PSS film with more fibrils of interconnected conductive chain (PEDOT) with 5\% DMSO doping or methanol treatment. TFMS-MeOH treatment packs the PEDOT chains more densely than polar solvent-treated does. The relatively low roughness of the height images and good smoothness of the phase images of TFMS-MeOH treated PEDOT:PSS film suggest the thin film with a higher density and a lower phase separation. Densely packed PEDOT chains increase the electrical conductivity to $\sim 3000 \mathrm{~S} \mathrm{~cm}^{-1}$. This implies that co-solvent treatment not only removes the PSSH acid produced in the treatment but also improves the alignment and densification of conductive polymer (PEDOT) chains. This might be the main reason why TFMS-MeOH treatment could improve the electrical conductivity without sacrificing other thermoelectric properties such as the Seebeck coefficient. The X-ray diffraction patterns of the PEDOT:PSS nanothin film with different treatments are presented in Fig. 5. The two distinct peaks at $2 \theta$ values of approximately at $3.5^{\circ}$ and $25.6^{\circ}$ in the pristine PEDOT:PSS film correspond to the lattice $d$ spacing of $25.2 \AA$ and $3.5 \AA$, calculated in terms of Bragg's law, $2 d \sin \theta=\lambda$. The $d$ spacing of 25.2 A observed at $2 \theta \approx 3.5^{\circ}$ can be assigned to the distance between the lamella stacking ( $d$ 100) due to the alternate ordering distance of PEDOT and PSS chain. This lamella stacking distance agrees well with the widths of the PEDOT and PSS

Table 1 Comparison of electric properties of PEDOT:PSS film with and without TFMS-MeOH treatment

\begin{tabular}{llcc}
\hline Sample & $\mu\left(\mathrm{cm}^{2} \mathrm{~V}^{-1} \mathrm{~s}^{-1}\right)$ & $n\left(\mathrm{~cm}^{-3}\right)$ & $\delta\left(\mathrm{S} \mathrm{cm}^{-1}\right)$ \\
\hline Pristine & $0.631 \pm 0.06$ & $3.86 \pm 0.12 \times 10^{18}$ & $0.69 \pm 0.1$ \\
MeOH & $0.684 \pm 0.05$ & $3.78 \pm 0.13 \times 10^{21}$ & $851 \pm 18$ \\
DMSO & $0.651 \pm 0.05$ & $4.63 \pm 0.1 \times 10^{21}$ & $898 \pm 20$ \\
TFMS-MeOH & $0.728 \pm 0.07$ & $1.451 \pm 0.25 \times 10^{22}$ & $2783 \pm 150$
\end{tabular}


Table 2 Thermoelectric properties of PEDOT:PSS film with and without TFMS-MeOH treatment

\begin{tabular}{|c|c|c|c|c|c|c|}
\hline Sample & $S\left(\mu \mathrm{V} \mathrm{K}^{-1}\right)$ & $\delta\left(\mathrm{S} \mathrm{cm}^{-1}\right)$ & 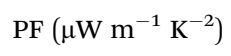 & $C_{\mathrm{p}}\left(\mathrm{J} \mathrm{K} \mathrm{g}^{-1}\right)$ & $\alpha\left(\mathrm{mm}^{2} \mathrm{~S}^{-1}\right)$ & $\kappa\left(\mathrm{W} \mathrm{K}^{-1} \mathrm{~m}^{-1}\right)$ \\
\hline Pristine & $17.6 \pm 1.5$ & $0.7 \pm 0.1$ & $0.0216 \pm 0.003$ & $2.36 \pm 0.05$ & $0.074 \pm 0.005$ & $0.279 \pm 0.030$ \\
\hline TFMS-MeOH & $21.9 \pm 2.0$ & $2980 \pm 150$ & $143 \pm 15$ & $1.87 \pm 0.05$ & $0.075 \pm 0.005$ & $0.224 \pm 0.020$ \\
\hline
\end{tabular}

chain, which are 7.5 and $15.5 \AA$, respectively, estimated according to chemical structural simulation. ${ }^{33}$ On the other hand, the $d$ spacing of $3.5 \AA$ spotted at $2 \theta \approx 25.6^{\circ}$ can be attributed to the distance between $\pi-\pi$ stacking $(d 010)$ of the PEDOT chains. After TFMS-MeOH treatment, there is slight change in the lamella stacking distance from 25.2 to $23.2 \AA$, and meanwhile the $\pi-\pi$ stacking distance marginally reduces from $3.5 \AA$ to $3.4 \AA$ (Fig. 5) The reduction in $\pi-\pi$ stacking distance suggests that PEDOT and PSS chains transform to quinoid structure from benzoid structure and therefore become more planar with TFMS-MeOH treatment. In addition, the peak associated with (010) plane decreases in comparison with (100) after TFMS-MeOH treatment, suggesting that such treatment shifts the orientation of PEDOT:PSS layer towards a more perpendicular direction with respect to the substrate. Compared with XRD characteristic peaks of the pristine PEDOT:PSS, the TFMS-MeOH treated film exhibits shaper diffraction peaks with the higher intensity in the low angle reflections $2 \theta$ at $3.8^{\circ}$ and $6.6^{\circ}$, corresponding separately to the lamella stacking distance $d(100)$ of two distinct alternate orderings of PEDOT and PSS chains, implying a higher crystallization degree of the PEDOT:PSS film. All the XRD results indicated that co-solvent treatment increased the lamella stacking between two assemblies and interchain coupling of PEDOT:PSS chains with more densely packed PEDOT, ${ }^{33,34}$ and increased the Seebeck coefficient of nanothin film through interface scattering. The Raman spectra of pristine and treated samples were examined and are shown in Fig. 6 . Treatment with $\mathrm{MeOH}$ and DMSO did not result in any significant changes in its main band at $1421 \mathrm{~cm}^{-1}$, which is assigned to the $C_{\alpha}=C_{\beta}$ stretching vibrations..$^{42,43}$ In contrast, with the treatments of TFMS-MeOH, the Raman band at $1421 \mathrm{~cm}^{-1}$ downshifted to $1387 \mathrm{~cm}^{-1}$, corresponding to a confirmation change from benzoid to quinoid structure of the PEDOT chains. ${ }^{42}$

Taking overall results together, we proposed the schematic diagram as illustrated in Fig. 7. Therefore, when PEDOT:PSS is treated with TFMS-MeOH co-solvent, the insulating polymer (PSS) was reacted with trifluoromethanesulfonic acid and washed out by methanol, respectively. While the conducting assemblies of PEDOT:PSS chains are aligned better and in turn become denser.

Table 1 give the electric properties of the pristine PEDOT:PSS film with treatment thin film by Hall effect measurements. The van der Pauw-Hall measurements was tested on a polycrystalline bar-shaped ingot with four coldpressed in contacts (one on each side of the ingot) positioned in a standard Hall geometry ${ }^{41}$ The pristine PEDOT:PSS film shows a lowest carrier concentration at room temperature of $n$ with $3.86 \pm 0.12 \times 10^{18} \mathrm{~cm}^{-3}$, indicative of p-type conduction and consistent with the positive Seebeck coefficient. The calculated conductivity was $0.69,851,898$, $2738 \mathrm{~S} \mathrm{~cm}^{-1}$ for pristine, mixing with $5 \%$ DMSO, methanol post-treatment and TFMS-MeOH post-treatment, respectively. In addition, carrier concentration $(n)$ increases significantly and mobility $(\mu)$ increases slightly after treatment. It is implied that the incorporation of prior results in the removal of the insulating PSS parts from PEDOT:PSS thin films, ${ }^{41,44,45}$ Table 2 summarizes the thermoelectric properties of PEDOT:PSS nanothin film measured before and after TFMS-MeOH treatment, including the Seebeck coefficient, electrical conductivity, power factor, thermal capacity, thermal diffusivity, and thermal conductivity. Similar to the Seebeck coefficient, the thermal conductivity of PEDOT:PSS was not much affected by the increase in electrical conductivity. The DMSO and $\mathrm{MeOH}$ treated films have a similar thermal conductivity of $0.25 \mathrm{~W}$ $\mathrm{m}^{-1} \mathrm{~K}^{-1}$ to the pristine PEDOT:PSS film. Based on the highest electrical conductivity of $2980 \mathrm{~S} \mathrm{~cm}^{-1}$ and the out-of-plane thermal conductivity $0.224 \mathrm{~W} \mathrm{~m}^{-1} \mathrm{~K}^{-1}$, the calculated power factor and $Z T$ value of TFMS-MeOH treated PEDOT:PSS nanothin film was $143 \mu \mathrm{W} \mathrm{m}^{-1} \mathrm{~K}^{-2}$ and 0.19 , respectively.

\section{Conclusions}

In conclusion, this anhydrous perfluorinated superacid and alcohol co-solvent post-treatment increased the electrical conductivity by effectively removing the insulating PSS parts and facilitating the formation of a more densely packed and a higher degree of crystalline structure as evidenced by the sharper XRD diffractions at the low angle of reflections as well as a shorter $\pi-\pi$ stacking distance. Upon TFMS-MeOH treatment, the electrical conductivity of the nanothin film achieved up to $2980 \mathrm{~S} \mathrm{~cm}^{-1}$, and Seebeck coefficient also reasonably increased to $21.9 \mu \mathrm{V} \mathrm{K}^{-1}$, leading to a power factor of $143 \mu \mathrm{W}$ $\mathrm{m}^{-1} \mathrm{~K}^{-2}$ and a $Z T$ of about 0.19 . The improvement of both electrical conductivity and Seebeck coefficient were the key strategies in the enhancement of TE performance of particularly for organic conductive polymers with the highly dense packing polymer chains. The post-treatment by this co-solvent consisting of superacid TFMS and methanol for conducting PEDOT:PSS film may open up a new potential in flexible thermoelectric power and sensor device.

\section{Conflicts of interest}

There are no conflicts to declare. 


\section{Acknowledgements}

The authors acknowledge support from the A*STAR, Industry Alignment Fund, Pharos "Hybrid thermoelectric materials for ambient applications” Program (Grant No. 1527200021).

\section{Notes and references}

1 G. J. Snyder and E. S. Toberer, Nat. Mater., 2008, 7, 105-114.

2 N. Dubey and M. Leclerc, J. Polym. Sci., Part B: Polym. Phys., 2011, 49, 467-475.

3 M. He, F. Qiu and Z. Lin, Energy Environ. Sci., 2013, 6, 13521361.

4 T. O. Poehler and H. E. Katz, Energy Environ. Sci., 2012, 5, 8110-8115.

5 O. Bubnova and X. Crispin, Energy Environ. Sci., 2012, 5, 9345-9362.

6 R. Yue, S. Chen, B. Lu, C. Liu and J. Xu, J. Solid State Electrochem., 2011, 15, 539-548.

7 N. Mateeva, H. Niculescu, J. Schlenoff and L. R. Testardi, J. Appl. Phys., 1998, 83, 3111-3117.

8 R. Yue and J. Xu, Synth. Met., 2012, 162, 912-917.

9 H. Kaneko, T. Ishiguro, A. Takahashi and J. Tsukamoto, Synth. Met., 1993, 57, 4900-4905.

10 Q. Zhang, Y. Sun, W. Xu and D. Zhu, Energy Environ. Sci., 2012, 5, 9639-9644.

11 Y. Y. Wang, J. Zhou and R. G. Yang, J. Phys. Chem. C, 2011, 115, 24418-24428.

12 G. H. Kim, L. Shao, K. Zhang and K. P. Pipe, Nat. Mater., 2013, 12, 719-723.

13 D. A. Mengistie, C. H. Chen, K. M. Boopathi, F. W. Pranoto, L. J. Li and C. W. Chu, ACS Appl. Mater. Interfaces, 2015, 7, 94-100.

14 C. Badre, L. Marquant, A. M. Alsayed and L. A. Hough, Adv. Funct. Mater., 2012, 22, 2723-2727.

15 J. Luo, D. Billet, T. Blaudeck, E. Sheremet, R. D. Rodriguez, D. R. Zahn, M. Toader, M. Hietschold, T. Otto and T. Gessner, J. Appl. Phys., 2014, 115, 054908.

16 J. S. Yeo, J. M. Yun, D. Y. Kim, S. Park, S.-S. Kim, M. H. Yoon, T. W. Kim and S. I. Na, ACS Appl. Mater. Interfaces, 2012, 4, 2551-2560.

17 Y. Xia and J. Ouyang, ACS Appl. Mater. Interfaces, 2010, 2, 474-483.

18 Y. Xia, K. Sun and J. Ouyang, Adv. Mater., 2012, 24, 24362440.

19 M. Vosgueritchian, D. J. Lipomi and Z. Bao, Adv. Funct. Mater., 2012, 22, 421-428.

20 J. Luo, D. Billep, T. Waechtler, T. Otto, M. Toader, O. Gordan, E. Sheremet, J. Martin, M. Hietschold and D. Zahn, J. Mater. Chem. A, 2013, 1, 7576-7583.

21 Q. Wei, M. Mukaida, K. Kirihara, Y. Naitoh and T. Ishida, Materials, 2015, 8, 732-750.

22 S. Ichikawa and N. Toshima, Polym. J., 2015, 47, 522-526.
23 J. Xiong, F. Jiang, H. Shi, J. Xu, C. Liu, W. Zhou, Q. Jiang, Z. Zhu and Y. Hu, ACS Appl. Mater. Interfaces, 2015, 7, 14917-14925.

24 S. Liu, H. Deng, Y. Zhao, S. Ren and Q. Fu, $R S C A d v ., 2015,5$, 1910-1917.

25 J. Xiong, F. Jiang, W. Zhou, C. Liu and J. Xu, RSC Adv., 2015, 5, 60708-60712.

26 S. H. Lee, H. Park, S. Kim, W. Son, I. W. Cheong and J. H. Kim, J. Mater. Chem. A, 2014, 2, 7288-7294.

27 N. Kim, S. Kee, S. H. Lee, B. H. Lee, Y. H. Kahng, Y. Jo, B. Kim and K. Lee, Adv. Mater., 2014, 26, 2268-2272.

28 T. C. Tsai, H. C. Chang, C. H. Chen, Y. C. Huang and W. T. Whang, Org. Electron., 2014, 15, 641-645.

29 U. Voigt, W. Jaeger, G. H. Findenegg and R. V. Klitzing, J. Phys. Chem. B, 2003, 107, 5273.

30 X. Crispin, S. Marciniak, W. Osikowicz, G. Zotti, A. W. Denier Van DerGon, F. Louwet, M. Fahlman, L. Groenendaal, F. De Schryver and W. R. Salaneck, J. Polym. Sci., Part B: Polym. Phys., 2003, 41, 2561.

31 B. J. Worfolk, S. C. Andrews, S. Parkc, J. Reinspach, N. Liua, M. F. Toney, S. C. B. Mannsfeldd and Z. Bao, PNAS, 2015, 112, 14138-14143.

32 N. Massonnet, A. Carella, A. de Geyer, J. F. Vincent and J. P. Simonato, Chem. Sci., 2015, 6, 412-417.

33 N. Kim, B. H. Lee, D. Choi, G. Kim, H. Kim, J. R. Kim, J. Lee, Y. H. Kahng and K. Lee, Phys. Rev. Lett., 2012, 109, 106405.

34 J. Zhou, E. Q. Li, R. Li, X. Xu, I. A. Ventura, A. Moussawi, D. H. Anjum, M. N. Hedhili, D. M. Smilgies, G. Lubineau and S. T. Thoroddsen, J. Mater. Chem. C, 2015, 11, 25282538.

35 J. Liu, L. Qiu, G. Portale, M. Koopmans, G. Ten Brink, J. C. Hummelen and L. J. A. Koster, Adv. Mater., 2017, 29, 1701641.

36 R. Kroon, D. Kiefer, D. Stegerer, L. Yu, M. Sommer and C. Muller, Adv. Mater., 2017, 29, 1700930.

37 A. M. Glaudell, J. E. Cochran, S. N. Patel and M. L. Chabinyc, Adv. Energy Mater., 2015, 5, 1401072.

38 L. Ye and Y. W. Mai, Polymer Blends and Polymer Composites, Key engineering Materials, 1997, vol. 137, ch. 2, p. 84.

39 H. K. Lee, J. Y. Kim and S. C. Kim, J. Membr. Sci., 1999, 163, 159-166.

40 H. Wang, U. Ail, R. Gabrielsson, M. Berggren and X. Crispin, Adv. Energy Mater., 2015, 5, 1500044.

41 Y.-J. Lin, W.-S. Ni and J.-Y. Lee, J. Appl. Phys., 2015, 117, 215501.

42 Z. Fan, P. Li, D. Du and J. Ouyang, Adv. Energy Mater., 2017, 7, 1602116.

43 M. Culebras, C. M. Gomez and A. Cantarero, J. Mater. Chem. A, 2014, 2, 10109.

44 Y. H. Kim, C. Sachse, M. L. Machala, C. May, L. MullerMeskamp and K. Leo, Adv. Funct. Mater., 2011, 21, 1076.

45 D. A. Mengistie, C. H. Chen, K. M. Boopathi, F. W. Pranoto, L. J. Li and C. W. Chu, ACS Appl. Mater. Interfaces, 2015, 7, 94-104. 